\title{
OPTICAL PROPERTIES OF NEW INDOTRICARBOCYANINE DYE AS A LIMITER OF LASER RADIATION POWER
}

\author{
V. A. Svetlichnyi, ${ }^{a}$ M. P. Samtsov ${ }^{b *}$ O. K. Bazyl, ${ }^{a}$ \\ O. V. Smirnov, ${ }^{\mathrm{a}}$ D. G. Mel'nikov, ${ }^{\mathrm{b}}$ and A. P. Lugovskii ${ }^{\mathrm{b}}$
}

UDC 535.37+535.34

We present results of experimental and theoretical studies of the optical characteristics of a new indotricarbocyanine dye that is capable of effectively limiting the power of laser radiation in the visible spectral range. The spectral-luminescent and energy characteristics of the dye molecules and their absorption spectra from the excited state with nanosecond resolution are investigated experimentally. Quantum-chemical methods are used to calculate electronic absorption spectra from the ground $\left(S_{0} \rightarrow S_{n}\right)$ and excited $\left(S_{1} \rightarrow S_{n}\right)$ states and to determine the nature of electronic states of the molecule and the rate constants of intramolecular photophysical processes. The results of the theoretical research agree with experimental data. It is shown that the investigated dye has singlet-singlet absorption at 400-600 $\mathrm{nm}$. Nonlinear absorption of the dye upon excitation by radiation of the second harmonic of a Nd:YAG laser is studied by z-scanning with an open diaphragm. The ratio of dye absorption cross sections from the excited and ground states at $532 \mathrm{~nm}$ is determined in the framework of a three-level model. The results are compared with those for previously studied compounds.

Key words: polymethine dye, spectral-luminescent properties, nonlinear absorption, quantum-chemical method.

Introduction. Polymethine dyes (PD) are broadly used in various branches of science and technology, e.g., in the photographic industry as photosensitizers [1], in biology and medicine as fluorescent markers and materials for photochemotherapy [2-4], in solar cells [5-7] and quantum electronics as passive gates, active media for tunable lasers, and nonlinear materials [8-10], etc. Recently the nonlinear optical properties of PD [11-15] have become especially interesting due to the search for new nonlinear materials for application as limiters of laser radiation power (nonlinear limiters) in the visible spectral region $[13,16,17]$. The properties of nonlinear optical materials based on organic dyes must satisfy several requirements in order to be highly efficient [18-21]. A high attenuation coefficient for laser radiation is one of the main parameters for optical limiters. In addition, there must be a low threshold and activation time, a broad dynamic range, high linear transmission in a broad spectral range, and, finally, a long operational lifetime for the active medium. PD in solutions satisfy most of these requirements. Therefore, the search for molecular structures in this class of organic molecules and comprehensive investigations of the optical properties of the most promising compounds used as the active medium of limiters are extremely timely.

The limiting properties of solutions of the indotricarbocyanine dye 2-\{7-[1,3,5-trimethyl-3-ethyl-2-(1H)-indoliniden]-4-chloro-3,5-trimethylene-1,3,5-heptatrien-1-yl\}-1,3,5-trimethyl-3-ethylindolium perchlorate (PD 7098) were previously studied upon excitation by nanosecond pulses of second harmonic radiation from a Nd:YAG laser (532 $\mathrm{nm}$ ) [21]. Quantum-chemical calculations and experimental investigations of the photophysical properties of this dye were carried out in order to elucidate the mechanism of nonlinear absorption. A model of nonlinear absorption was proposed. Quantitative estimates were made.

Materials and Methods. Quantum-chemical calculations. Quantum-chemical programs based on a semi-empirical method of partial neglect of differential overlap (PNDO) with the original parameter set [22] were used to cal-

*To whom correspondence should be addressed.

${ }^{\mathrm{a}}$ Siberian Physicotechnical Institute of Tomsk State University, Tomsk, Russia; bA. N. Sevchenko Research Institute for Applied Physics Problems of the Belarussian State University, 7 Kurchatov St., Minsk, 220064, Belarus, email: samtsov@bsu.by. Translated from Zhurnal Prikladnoi Spektroskopii, Vol. 74, No. 4, pp. 473-480, July-August, 2007. Original article submitted November 3, 2006. 
culate electronic absorption spectra of PD 7098 from the ground and first excited singlet states. The principal value of the used program set compared with existing ones is the ability to calculate rate constants of radiationless deactivation processes of excitation energy in the molecules. Comparison of the efficiency of various channels for energy dissipation in a multiatomic molecule can make its spectral-luminescent properties understandable. An examination of absorption spectra from excited states can establish what excited states participate in their formation and evaluate the possibility of populating states that are the initial ones for generation of these spectra.

Although the results of calculations of electronic transition characteristics were obtained for isolated molecules, i.e., without considering the solvent effect, they describe rather well the $S_{0} \rightarrow S_{n}$-absorption and satisfactorily the $S_{1}$ $\rightarrow S_{n}$-absorption spectra. This is due to the fact that the parameter system used in the calculations considers partially the universal intermolecular interaction.

Spectral-luminescent properties. Electronic absorption spectra were recorded using a PV1251 spectrophotometer and a CM2203 spectrofluorimeter with a spectrophotometer function. Fluorescence spectra and quantum yield of the dye were investigated using Fluorolog and CM2203 spectrofluorimeters. The fluorescence quantum yield $(\eta)$ was determined by a relative method using solutions of Nile Blue in ethanol $(\eta=0.23$ [23]) and HITCI in ethanol $(\eta=0.28$ [24]) as standards. The degree of polarization of the fluorescence $(P)$ of the dye was measured at $15^{\circ} \mathrm{C}$ using the Fluorolog spectrofluorimeter. The quantum yield of generation $(B)$ of singlet oxygen $\left({ }^{1} \mathrm{O}_{2}\right)$ was found by comparing the emission efficiency of ${ }^{1} \mathrm{O}_{2}$ in deuterochloroform upon photosensitization by the investigated dye and HITCI as a standard $(B=3.6 \%$ [25]). Luminescence of singlet oxygen in the $1-2 \mu \mathrm{m}$ spectral range was recorded on the laboratory apparatus described in the literature [26]. Radiation from a semi-conducting laser with $\lambda_{\text {gen }}=740.7 \mathrm{~nm}$ was used for excitation. The fluorescence quenching kinetics of the dye were analyzed using a pulsed spectrofluorimeter developed in the Spectroscopy Laboratory of A. N. Sevchenko NII PFP (Minsk, Belarus) [27]. The experimental uncertainty of the fluorescence lifetime $\Delta \tau= \pm 0.1 \mathrm{~ns}$; of the degree of fluorescence polarization, $\pm 0.4 \%$. The uncertainty in the fluorescence quantum yield was less than $15 \%$. Measurements were made using thermostatted samples because the PD luminescence characteristics are highly temperature dependent.

Absorption spectra from the excited state. Absorption spectra from the excited states of PD 7098 solutions were obtained by pulsed flash photolysis with nanosecond resolution. The apparatus on which the measurements were made has been described in detail [28].

Induced nonlinear absorption was investigated in the spectral range 350-650 nm. Excitation was carried out at two wavelengths, $308 \mathrm{~nm}$ (XeCl-laser, $\tau_{\text {pul }}=12 \mathrm{~ns}, E_{\text {pul }}=40 \mathrm{~mJ}$ ) and $690 \mathrm{~nm}$ (Oxazine 1 dye laser, $\tau_{\text {pul }}=10 \mathrm{~ns}$, $\left.E_{\text {pul }}=18 \mathrm{~mJ}\right)$. The optical delay line $(0-40 \mathrm{~ns})$ enabled absorption induced in the medium by the laser pulse to be recorded during the excitation pulse and several nanoseconds after it was finished. This enabled short-lived singlet-singlet and long-lived absorption (e.g., due to photoproducts, triplet-triplet transitions, etc.) to be separated.

Absorption cross section from the excited state. The absorption cross section $\sigma_{1 n}$ at the wavelength of second harmonic radiation from a Nd:YAG laser $(532 \mathrm{~nm})$ was determined by a single-beam method of $z$-scanning with an open diaphragm that consisted of investigating transmission of a cuvette with dye solution as a function of its position relative to the lens focal plane [29]. The apparatus parameters (time and space-energy characteristics of the exciting radiation) for $z$-scanning have been published [15]. The maximum power density created in the focal plane was 750 $\mathrm{MW} / \mathrm{cm}^{2}$. A spherical lens $(F=100 \mathrm{~mm})$ was used for focusing. An ethanol solution of the dye was investigated in a 1-mm quartz cuvette. The linear absorption coefficient of the medium at the wavelength of the exciting radiation was less than $0.05 \mathrm{~cm}^{-1}$.

Limitation of laser radiation power. We investigated laser radiation transmission as a function of power density for excitation by second harmonic radiation from a LQ-129 Nd:YAG laser (Solar LS, $\tau_{\text {pul }}=15 \mathrm{~ns}, E_{\text {pul }}=100$ $\mathrm{mJ}, \lambda_{\max }=532 \mathrm{~nm}$ ). This same laser was used for the $z$-scanning experiment. Radiation was focused by a long-focus lens $(f=600 \mathrm{~mm})$ into the cuvette with the studied solution $(5 \mathrm{~mm}$ thick). The cuvette was located in front of the lens focal plane in convergent beams. Areas of the beam transverse cross section at the entrance and exit of the cuvette varied by less than 5\%. Linear transmittance of the solution (measured on the spectrophotometer) was $70-80 \%$. The radiation power density incident on the cuvette was measured in the range $0.1-150 \mathrm{MW} / \mathrm{cm}^{2}$. Radiation attenuation was carried out with nonselective calibrated light filters. Energy characteristics were recorded with an IMO-2H calorimeter and an ED-100a Gentec EO pyroelectric detector. 


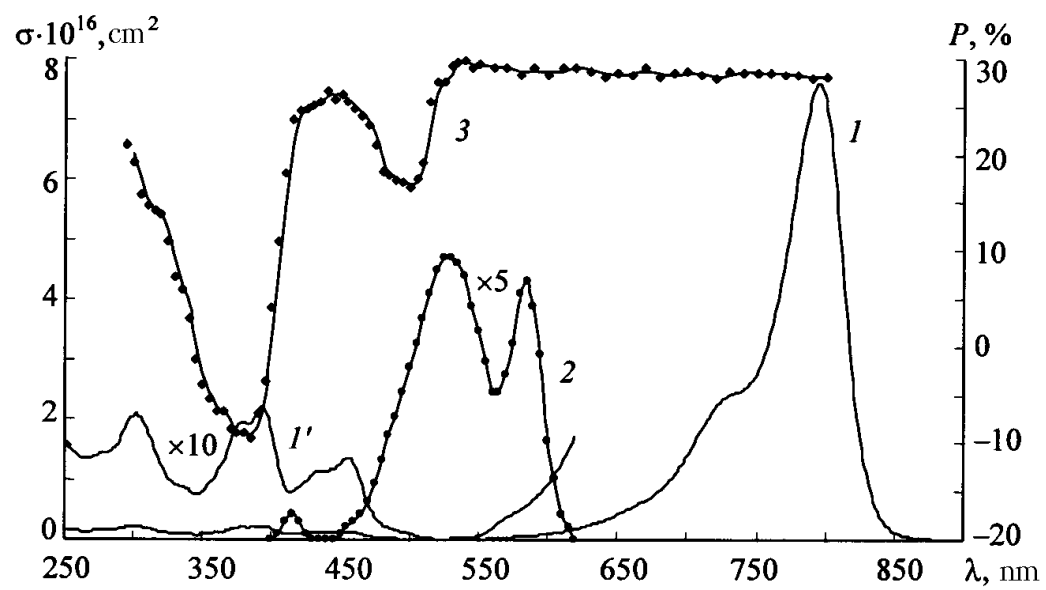

Fig. 1. Absorption spectra from the ground $\left(1,1^{\prime}\right)$ and excited (2) states and polarization spectrum at $15^{\circ} \mathrm{C}$ and plots at the fluorescence band maximum (3) of PD 7098 in ethanol.

Results and Discussion. Linear spectral-luminescence properties. The absorption spectrum of PD 7098 in ethanol (Fig. 1, curve 1) contains a strong $\left(\sigma_{01}=7.6 \cdot 10^{-16} \mathrm{~cm}^{2}\right)$ long-wavelength absorption band with a maximum near $790 \mathrm{~nm}$. Absorption of the investigated dye in the UV and visible regions (350-630 nm) is less than $2.2 \cdot 10^{-17}$ $\mathrm{cm}^{2}$. At $532 \mathrm{~nm}$, the absorption cross section is only $1.5 \cdot 10^{-18} \mathrm{~cm}^{2}$, which is one of the conditions for using this compound for limitation of optical radiation power in the visible region. The absorption spectrum in the range 250-620 nm (Fig. 1, curve 1') is magnified by 10 times for clarity. Dye in ethanol fluoresces with quantum yield $\eta=7 \%$. The fluorescence maximum is located at $815 \mathrm{~nm}$. The half-width of the spectrum is $43 \mathrm{~nm}$. The fluorescence decay kinetics of the dye are single exponential. The fluorescence lifetime is $0.7 \mathrm{~ns}$. The transmission rate constant from the results is $k_{\mathrm{r}}=10^{8} \mathrm{~s}^{-1}$.

Polarized spectra of the dye were investigated in order to assign absorption bands to particular electronic transitions in it. Because the fluorescence lifetime of the dye is rather short, the degree of fluorescence polarization upon excitation at the longest wavelength absorption band and $15^{\circ} \mathrm{C}$ reached $29 \%$. The degree of fluorescence polarization was constant upon excitation within the long-wavelength absorption band. A polarization minimum (Fig. 1, curve 3) was observed in the short-wavelength region upon excitation at $\sim 490 \mathrm{~nm}$. Decreasing the wavelength of the exciting light further caused oscillations of the degree of fluorescence polarization and produced correlations in the positions of the dye polarization spectrum extrema and the absorption band maxima (Fig. 1).

The quantum yield of ${ }^{1} \mathrm{O}_{2}$ generation was determined by recording luminescence of singlet oxygen upon excitation of PD 7098 and HITCI in $\mathrm{CDCl}_{3}$ by radiation with $\lambda=740.7 \mathrm{~nm}$. For PD $7098, B=0.37 \%$. As shown before [30], the main mechanism of photosensitization of oxygen by tricarbocyanine dyes is radiationless energy transfer from the dye in the triplet state. Therefore, $B$ may characterize the yield of intercombinational conversion of dye molecules. The intercombinational conversion rate constant of dye, $k_{\mathrm{ST}}=5 \cdot 10^{6} \mathrm{~s}^{-1}$, was estimated from the experimental results.

Electronic absorption spectra of the dye and data from polarization measurements were considered during quantum-chemical calculation of the spectra of electronically excited states.

The following molecular geometry of PD 7098 was used for the modeling. The dye molecule has point symmetry $C_{2 v}$. Because there are at present no experimental data for the structure of PD 7098, average bond lengths and angles were used [31]. The terminal fragments were taken to be planar and situated in the $X Y$ plane of the molecule. The $\mathrm{C}$ atoms of the polymethine chain deviated from the plane of the terminal fragments by less than $0.3 \AA$. $\mathrm{C}$ atoms of the polymethine chain that were bonded to the central fragment were located under the plane of the terminal fragments whereas $\mathrm{C}$ atoms bonded to these fragments were above this plane. Such a structure for the polymethine chain gives calculated electronic transition intensities that agree best with the experimental absorption spectrum from the ground state. 

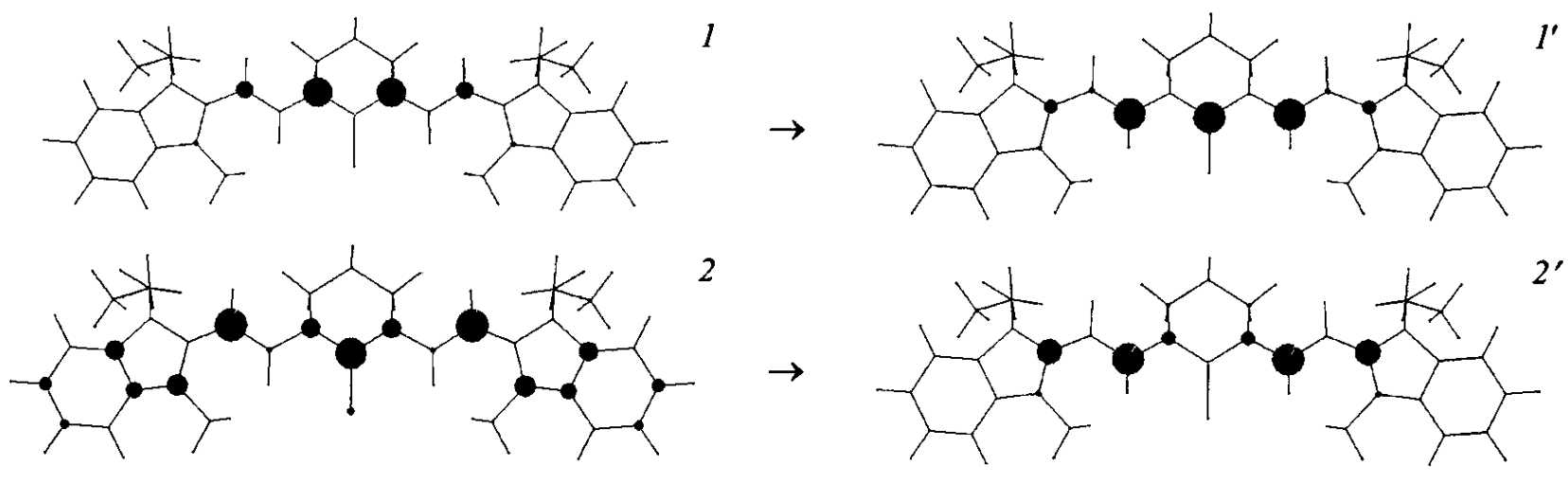

Fig. 2. Molecular diagrams of the two highest occupied $(1,2)$ and the two lowest unoccupied $\left(1^{\prime}, 2^{\prime}\right)$ molecular orbitals. Circles show the contribution of atomic-orbital wave functions to the formation of molecular orbitals.

TABLE 1. Calculated Frequencies (v) and Oscillator Strengths $(f)$ of Electronic Transitions from the Ground State for Dye PD 7098 and Experimental Data

\begin{tabular}{|c|c|c|c|c|c|c|c|}
\hline \multirow{2}{*}{\multicolumn{5}{|c|}{ Calculation }} & \multicolumn{3}{|c|}{ Experiment } \\
\hline & & & & & \multirow{2}{*}{$\begin{array}{c}\text { toluene } \\
\lambda_{\max } \\
\mathrm{nm}\end{array}$} & \multicolumn{2}{|l|}{ ethanol } \\
\hline$S_{0} \rightarrow S_{n}$ & $\begin{array}{l}v\left(S_{0} \rightarrow S_{n}\right) \\
\mathrm{cm}^{-1}\end{array}$ & $\begin{array}{c}\lambda\left(S_{0} \rightarrow S_{n}\right) \\
\mathrm{nm}\end{array}$ & $f\left(S_{0} \rightarrow S_{n}\right)$ & $\begin{array}{c}P \\
\left(S_{0} \rightarrow S_{n}\right)\end{array}$ & & $\begin{array}{c}\Delta \lambda \text { of extremes } P, \\
\mathrm{~nm}\end{array}$ & $\begin{array}{c}\lambda_{\max }, \\
\mathrm{nm}\end{array}$ \\
\hline$S_{1}(\pi \pi)$ & 13,291 & 752 & 0.918 & $X$ & 778 & $530-800(X)$ & 793 \\
\hline$S_{2}(\pi \pi)$ & 21,245 & 471 & 0.014 & $Y$ & 490 & $480-505(Y)$ & 490 \\
\hline$S_{3}(\pi \pi)$ & 26,274 & 381 & 0.073 & $X$ & 424,442 & $415-460(X)$ & 425,452 \\
\hline$S_{4}(\pi \pi)$ & 26,295 & 379 & 0.052 & $Y$ & & & \\
\hline$S_{5}(\pi \pi)$ & 27,991 & 357 & 0.318 & $\mathrm{X}$ & 376,390 & $370-385(Y)$ & 375,389 \\
\hline $\mathrm{S}_{6}(\pi \pi)$ & 28,762 & 348 & 0.052 & $Y Z$ & & & \\
\hline$S_{7}(\pi \pi)$ & 32,198 & 311 & 0.003 & $X$ & & & \\
\hline$S_{8}(\pi \pi)$ & 32.592 & 307 & 0.036 & $Z$ & 310 & $290-300(X)$ & 298 \\
\hline$S_{9}(\pi \pi)$ & 34,551 & 289 & 0.028 & $X$ & & & \\
\hline
\end{tabular}

Comparison of the calculated and experimental $S_{0} \rightarrow S_{n}$ spectra indicates that the calculated energies of the electronic transitions, especially of the lowest excited singlet states, agree well with the experimental ones (Table 1). This suggests that the calculation corresponds with the experiment and makes it possible to predict induced absorption spectra from the lowest excited states.

The calculation indicates that the long-wavelength absorption band of PD consists of a single electronic transition, $S_{0} \rightarrow S_{1}\left(\pi \pi^{*}\right)$ that is polarized along the long axis $(X)$ of the molecule. This result agrees with the constant degree of fluorescence polarization upon excitation into the long-wavelength absorption band of the dye. The electronic transition corresponding to this band consists $\sim 80 \%$ of a singly excited configuration between the highest occupied 1 and the lowest unoccupied $1^{\prime}$ molecular orbitals (MO) and $\sim 20 \%$ of a singly excited configuration between the 2 and $2^{\prime}$ orbitals (Fig. 2). The spatial localization of the MOs forming the long-wavelength absorption band in the PD spectrum suggests that the terminal fragments of the molecule participate little in the formation of the long-wavelength absorption. An analysis of the MOs forming the PD absorption spectrum in the range up to $30,000 \mathrm{~cm}^{-1}$ indicates that atomic oribtals (AO) of the terminal fragments participate in electronic transitions into higher excited states. However, there are no transitions connected only with the terminal fragments without involvement of the AO of the polymethine 


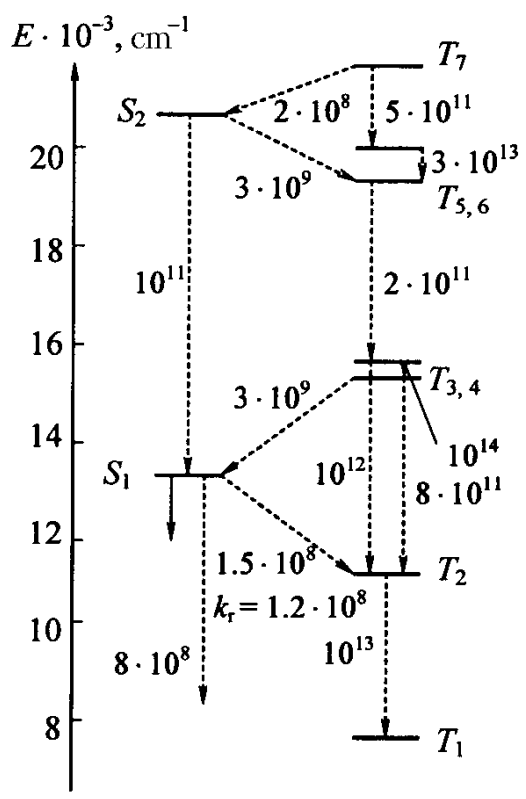

Fig. 3. Energy diagram of lowest electronically excited states of PD 7098. Solid and dashed lines correspond to radiative and nonradiative deactivation processes of excitation energy.

chain. For example, MOs formed by AO of terminal fragments but with a contribution of $\mathrm{C}$ AO of the polymethine chain participate in formation of $S_{2}\left(\pi \pi^{*}\right)$ states. The calculations agree with conclusions that were made earlier based on experimental investigations of the nature of the short-wavelength absorption bands and polarized spectra of symmetric PD [32]. It should be noted that the energy gap between separate electronic states decreases as the energy of the electronically excited states increases. This leads to their spectral overlap. The electronic states form a practically continuous spectrum in the region above $\sim 32,000 \mathrm{~cm}^{-1}$.

Table 1 shows that the calculation and experiment agree not only in the position of the maxima forming the absorption bands and their intensities but also in the polarization. This makes the interpretation of the spectra from the calculations more reliable. Comparison of the calculated polarization directions of the electronic transitions (Table 1) and the change of the degree of fluorescence polarization in the experimental spectrum is consistent with the correspondence between the calculation and experiment. Thus, changes in the polarized spectrum in the spectral ranges 500, 380 , and $330 \mathrm{~nm}$ are related to the $S_{0} \rightarrow S_{2}, S_{0} \rightarrow S_{4}$, and $S_{0} \rightarrow S_{6}$ transitions, which are polarized in the plane of the molecule along the short axis $(Y)$.

Figure 3 shows the calculated energy diagram of the electronically excited states of PD 7098. The calculations indicate that radiationless relaxation of the molecule into the $S_{1}$ state occurs within $\sim 10^{-11} \mathrm{~s}$ after the transition into higher lying excited states. The geometry of the molecule in the $S_{1}$ state changes insignificantly, which is consistent with the small Stokes shift of the fluorescence band of $\sim 400 \mathrm{~cm}^{-1}$ (experimental values). The lowest excited singlet state decays mainly through internal conversion. The calculated fluorescence rate constant, $k_{\mathrm{r}}=1.2 \cdot 10^{8} \mathrm{~s}^{-1}$, corresponds with the experimental value, $k_{\mathrm{r}}=10^{8} \mathrm{~s}^{-1}$.

A marked difference is observed between the calculated and experimental values (more than an order of magnitude) for the rate constant of intercombinational conversion. This may be due to an elevated calculated value of $k_{\mathrm{ST}}$ as a result of the lack of experimental data for the position of the lowest triplet state of PD 7098. This leads to uncertainty in the determination of the energy of the $T_{1}$ and $T_{2}$ states.

Nonlinear absorption. Low-intensity linear absorption of the dye in the visible region makes it easy to study absorption from excited states. Figure 1 (curve 2) shows a magnified (5×) induced absorption (IA) spectrum for an ethanol solution of PD 7098 upon excitation by nanosecond radiation at $690 \mathrm{~nm}$ from a dye laser (excitation into the $S_{1}$ state). The pulse of the probing radiation coincided in time with the exciting pulse. There was no IA in the me- 

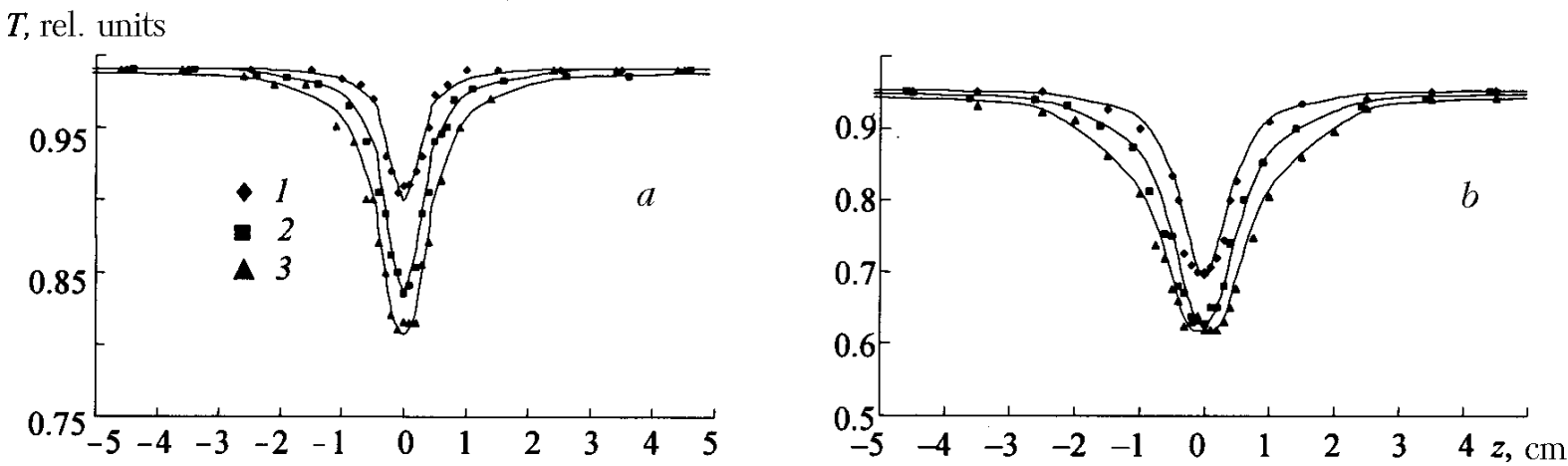

Fig. 4. Transmittance as a function of cuvette $(a=1 \mathrm{~mm})$ position relative to the lens focal plane for PD 7098 (a) and PD 823 (b) in ethanol (dye concentration $10^{-4} \mathrm{M}$ ) upon excitation by nanosecond pulses with energy 0.07 (1), 0.18 (2), and $0.35 \mathrm{~mJ}(3)$; solid lines are approximations for a three-level diagram.

TABLE 2. Calculated Frequencies ( $v)$ and Oscillator Strengths $(f)$ of Electronic Transitions from the $S_{1}$ State for Dye PD 7098 and Experimental Data

\begin{tabular}{|c|c|c|c|c|c|}
\hline \multirow{2}{*}{$S_{n}$} & \multicolumn{3}{|c|}{ Calculation } & \multicolumn{2}{c|}{ Experiment (ethanol) } \\
\cline { 2 - 6 } & $\lambda\left(S_{1} \rightarrow S_{n}\right), \mathrm{nm}$ & $v\left(S_{1} \rightarrow S_{n}\right), \mathrm{cm}^{-1}$ & $f\left(S_{1} \rightarrow S_{n}\right)$, & $\lambda, \mathrm{nm}$ & $v, \mathrm{~cm}^{-1}$ \\
\hline$S_{4}$ & 763 & 13,104 & 0.058 & & \\
$S_{6}$ & 646 & 15,470 & 0.443 & 585 & 17,100 \\
$S_{7}$ & 529 & 18,907 & 0.010 & & 19,250 \\
$S_{8}$ & 518 & 19,300 & 0.021 & 520 & \\
$S_{11}$ & 460 & 21,748 & 0.021 & & \\
$S_{12}$ & 442 & 22,630 & 0.030 & & \\
$S_{13}$ & 439 & 22,800 & 0.013 & & \\
$S_{15}$ & 428 & 22,340 & 0.176 & & \\
$S_{18}$ & 418 & 23,900 & 0.045 & & \\
\hline \hline
\end{tabular}

dium if the probing signal was delayed by $40 \mathrm{~ns}$ relative to the exciting pulse. Thus, the observed IA is short-lived and is probably due to $S_{1} \rightarrow S_{n}$ transitions. The IA spectrum upon excitation by a radiation pulse from a XeCl laser at $308 \mathrm{~nm}$ (near the 7th and 8th excited singlet states of the molecule) with a pulse length of $12 \mathrm{~ns}$ is similar to the spectrum shown in Fig. 1 (curve 2). This confirms the calculation of the fast radiationless relaxation of energy absorbed into the $S_{1}$ state. Analogous behavior was observed for IA spectra of PD studied before [15, 21]. The IA maximum coincides with the wavelength of second harmonic radiation from a Nd:YAG laser.

It should be noted that the experimental IA spectrum is distorted by partial overlap of bands due to $S_{1} \rightarrow S_{n}$ and $S_{0} \rightarrow S_{n}$ transitions, especially in the long-wavelength region where the $S_{0} \rightarrow S_{1}$ transition starts to appear. The population of the $S_{1}$ state for the exciting pulse parameters and excitation power density $\left(25 \mathrm{MW} / \mathrm{cm}^{2}\right)$ used in the experiment is less than several percent. Reducing the power density to $10 \mathrm{MW} / \mathrm{cm}^{2}$ halves the intensity of the longwavelength IA band and shifts it by $15 \mathrm{~nm}$ to higher energy. Thus, the intensity of the IA band may be much greater and its maximum shifted to longer wavelengths because of overlap with the absorption spectrum from the ground state.

Table 2 gives the calculated $S_{1} \rightarrow S_{n}$ absorption compared with the experimental values. The strongest transition, $S_{1} \rightarrow S_{6}$, lies near $646 \mathrm{~nm}$ and overlaps the absorption spectrum of the molecule from the ground state. Apparently we observed also the experimental $S_{1} \rightarrow S_{6}$ absorption band edge near $585 \mathrm{~nm}$. The short-lived IA in the spectral region below $550 \mathrm{~nm}$ is due to several low-intensity singlet-singlet transitions. It can be observed only due to the negligibly small absorption from the ground state in this spectral region. 


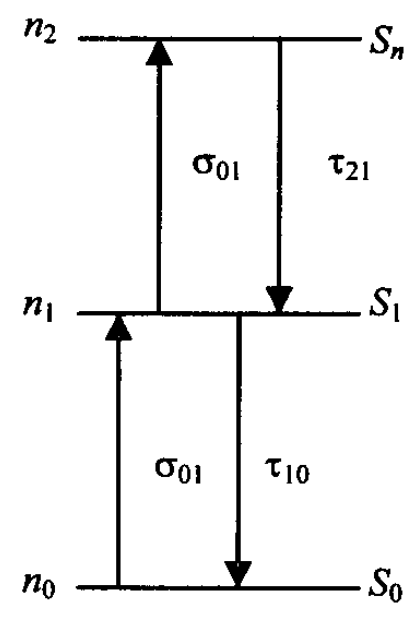

Fig. 5. Three-level diagram of photoprocesses in polymethine dye.

It should be noted in general that the quantitative agreement of the calculated and experimental spectra for singlet-singlet IA is worse than for absorption from the ground state. There are two main reasons for this. These are the distortion of the experimental IA spectra (influence of absorption from the ground state on the position of the long-wavelength $S_{1} \rightarrow S_{n}$ absorption band) and the less accurate determination of the energy of the higher excited states (compared with $S_{1}$ ) by quantum-chemical methods.

Determination of absorption cross section from $S_{1}$ state by $z$-scanning method. A single-beam $z$-scanning method with an open diaphragm was used for quantitative determination of the absorption intensity from the excited state (the ratio of absorption cross sections in the ground and excited states $k=\sigma_{1 n} / \sigma_{01}$ is an important parameter of optical limiters) at a single wavelength [15, 29]. Figure 4 shows the relative transmission of an ethanol solution of PD 7098 as a function of the position of the cuvette relative to the lens focal plane for three different exciting pulse energies and the analogous plots for PD 823 that was studied previously [15].

The absorption cross section in the excited state at the pumping wavelength $(532 \mathrm{~nm})$ can be determined from the $z$-scanning data. For this, we use a three-level diagram (Fig. 5) that is described by the system of equations

$$
\begin{gathered}
\frac{\partial n_{0}}{\partial t}=-\frac{\sigma_{01} I}{h \nu} n_{0}+\frac{n_{1}}{\tau_{10}}, \\
\frac{\partial n_{1}}{\partial t}=\frac{\sigma_{01} I}{h \nu} n_{0}-\frac{\sigma_{1 n} I}{h \nu} n_{1}-\frac{n_{1}}{\tau_{10}}+\frac{n_{2}}{\tau_{21}}, \\
\frac{\partial n_{2}}{\partial t}=\frac{\sigma_{1 n} I}{h \nu} n_{1}-\frac{n_{2}}{\tau_{21}},
\end{gathered}
$$

where $n_{0}, n_{1}$, and $n_{2}$ are the populations of the $S_{0}, S_{1}$, and $S_{n}$ states; $\sigma_{01}$ and $\sigma_{1 n}$ are the absorption cross sections from the $S_{0}$ and $S_{1}$ states; and $\tau_{01}$ and $\tau_{21}$ are the lifetimes of the $S_{0}$ and $S_{n}$ states.

Taking into account features of PD photophysical properties for the employed three-level diagram, the following assumptions are made.

1) Because the main channel for dissipating energy for the investigated dyes is internal conversion and the intercombinational conversion rate constant is several orders of magnitude less than the transition rate with emission from the $S_{1}$ state (with a fluorescence quantum yield of 7\%), triplet states of the molecules are not considered.

2) Since forced emission of the dye solutions was not observed in our experiments at the used concentrations $\left(\sim 10^{-4} \mathrm{M}\right)$, initial transmittance $T_{0}=95-99 \%$, excited volume in the $z$-scanning experiment, fluorescence quantum yield of $7 \%$, and rather intense IA at the excitation wavelength, the change of lifetime of the $S_{1}$ state as a result of forced emission of the dye was not considered. 
The system of equations (1)-(3) was solved numerically and the transmittance of the medium at the given intensity of the exciting pulse radiation was determined:

$$
\frac{\partial I}{\partial z}=-\alpha I=-\left(\sigma_{0} n_{0}+\sigma_{1} n_{1}\right) I .
$$

The variable, besides the absorption cross section from the $S_{1}$ state, is the lifetime of higher lying $S_{n}$ states that was determined preliminarily from the quantum-chemical calculations.

Figure 4 gives the modeling results. The best agreement of the experimental data with the modeling results for the medium transmittance as a function of intensity of exciting radiation is observed for $k=64 \pm 4$ and $\tau_{21}=$ $4-6 \cdot 10^{-12} \mathrm{~s}$ for PD 7098 and $k=18 \pm 2$ and $\tau_{21}=2-4 \cdot 10^{-12} \mathrm{~s}$ for PD 823 . The results for $k$ correspond to the ratio of limiting ability of these dyes. For PD 7098, for which the ratio of absorption cross sections in the ground and excited states is greater, the attenuation coefficient (AC) is also greater. The AC is equal to the ratio of linear transmission to transmission with a given excitation power density. The $\mathrm{AC}$ at $100 \mathrm{MW} / \mathrm{cm}^{2}$ excitation power density and linear transmission $T_{0}=70 \%$ is 22 for PD 7098 and 9.5 for PD 823 .

Numerical modeling of the transmittance as a function of incident radiation power density showed that a power density of hundreds of $\mathrm{MW} / \mathrm{cm}^{2}$ with nanosecond excitation creates a high population of not only $S_{1}$ but also higher lying singlet states (in our instance the $S_{2}$ state, the lifetime of which, according to quantum-chemical calculations, is greatest for higher lying states). The population of the $S_{2}$ state at the maximum excitation power density used in our experiments $\left(750 \mathrm{MW} / \mathrm{cm}^{2}\right)$ can reach $40 \%$ according to modeling results.

The resulting ratios of absorption cross sections in the excited and ground states are greater than those determined previously (20-25 for PD 7098 [16] and 11 for PD 823 [15]). We note that the differences increase if $k$ is increased. This is due to the fact that the previous calculations $[15,16]$ used simplified formulas that did not consider saturation of the $S_{1}$ state at large intensities of exciting radiation and the possibility of significant population of higher lying singlet states.

Conclusion. Calculations of electronic absorption spectra from the ground $\left(S_{0} \rightarrow S_{n}\right)$ and excited $\left(S_{1} \rightarrow S_{n}\right)$ states agree with experimental results. The nature of the electronic states is established. Rate constants of intramolecular photophysical processes are determined. It is shown that the observed short-lived nonlinear absorption of the dye in the spectral range $480-590 \mathrm{~nm}$ is due to $S_{1} \rightarrow S_{n}$ transitions in the molecule. Nonlinear absorption is investigated using the $z$-scanning method with an open diaphragm. The ratio of dye absorption cross sections from $S_{1}$ and ground states at $532 \mathrm{~nm}$ is determined within the framework of a three-level diagram and is $k=64 \pm 4$. The completed investigations indicate that limitation of laser radiation power by this dye is due to reversible saturated absorption in singlet state channels.

Further experimental and theoretical investigations of molecules with a relatively long lifetime for higher lying excited states are planned because the modeling results for nonlinear absorption showed a possible significant population of higher lying excited states for polymethine dyes.

Acknowledgments. We thank V. Ya. Artyukhov for help and useful comments regarding the quantum-chemical investigations. The work was performed with partial financial support of INTAS (project 03-50-5765), the Russian Foundation for Basic Research (grant 07-02-00155-a), the joint Russian-American program BRHE (grant Y2-P-16-01), and the Belarus Republic Foundation for Basic Research.

\section{REFERENCES}

1. T. James, The Theory of the Photographic Process, 4th Ed., Macmillan, New York (1977).

2. E. S. Voropai, M. P. Samtsov, V. N. Chalov, and É. A. Zhavrid, Zh. Prikl. Spektrosk., 68, No. 3, 359-362 (2001).

3. G. Patonay, J. Salon, J. Sowell, et al., Molecules, 9, 40-49 (2004).

4. U. Schobel, H. Egelhaaf, D. Fruhlich, et al., J. Fluoresc., 10, No. 2, 147-154 (2000).

5. X. Chen, J. Guo, X. Peng, M. Guo, et al., J. Photochem. Photobiol. A: Chem., 171, 231-236 (2005).

6. F. Naesch, G. Tornare, L. Zuppiroli, et al., Sol. Energy Mater. Sol. Cells, 87, 817-824 (2005).

7. M. Matsu, Y. Hashimoto, K. Funabiki, et al., Synth. Met., 148, 147-153 (2005). 
8. V. I. Bezrodnyi and A. A. Ishchenko, Opt. Laser Technol., 34, 7-13 (2002).

9. J. Zhang, W. Luo, and Z. Zhu, Dyes Pigm., 28, No. 1, 1-6 (1995).

10. Z. Dai, B. Peng, Q. Yang, X. Liu, and P. Ye, Chem. Phys. Lett., 317, 9-12 (2000).

11. R. S. Lepkowicz, C. M. Cirloganu, J. Fu, et al., J. Opt. Soc. Am. B: Opt. Phys., 22, No. 12, $2664-2685$ (2005).

12. Y. H. Meyer, M. Pittman, and P. Plaza, J. Photochem. Photobiol. A: Chem., 114, 1-21 (1998).

13. D. A. Oulianov, A. S. Dvornikov, and P. M. Rentzepis, Opt. Commun., 205, 427-436 (2002).

14. J. Fu, O. V. Przhonska, L. A. Padilha, D. J. Hagan, et al., Chem. Phys., 321, 257-268 (2006).

15. V. A. Svetlichnyi, Kvantovaya Elektron. (Moscow), 36, No. 1, 51-55 (2006).

16. T. N. Kopylova, A. P. Lugovskii, V. M. Podgaetskii, O. V. Ponomareva, and V. A. Svetlichnyi, Kvantovaya Elektron. (Moscow), 36, No. 3, 274-279 (2006).

17. J. Lim, O. Przhonska, S. Khodja, S. Yang, et al., Chem. Phys., 245, $79-97$ (1999).

18. R. Lepkowicz, O. Przhonska, J. Hales, et al., Chem. Phys., 286, 277-291 (2003).

19. R. Lepkowicz, C. Cirloganu, O. Przhonska, et al., Chem. Phys., 306, 171-183 (2004).

20. H. Gratz, A. Penzkofer, C. Abels, et al., J. Photochem. Photobiol. A: Chem., 128, 101-109 (1999).

21. V. A. Svetlichnyi, N. N. Svetlichnaya, E. I. Sichenko, E. A .Vaitulevich, and I. N. Lapin, Opt. Atmos. Okeana, 16, No. 8, 747-750 (2003).

22. V. Ya. Artyukhov and A. I. Galeeva, Izv. Vyssh. Uchebn. Zaved., Fiz., 11, 96-100 (1986).

23. C. A. Parker, Photoluminescence of Solutions with Applications to Photochemistry and Analytical Chemistry, Elsevier, New York (1968).

24. B. I. Stepanov, ed., Catalog of active laser media based on solutions of organic dyes and related compounds, Inst. Fiz. Akad. Navuk BSSR, Minsk (1977), 162.

25. E. S. Voropai and M. P. Samtsov, Opt. Spektrosk., 82, 577-580 (1997).

26. V. Tkachov and A. Mudryi, Just. Phys. Conf. Ser. 31, Pergamon Press, London-Bristol (1977), 231.

27. E. S. Voropai, M. P. Samtsov, K. N. Kaplevskii, et al., Vestn. Beloruss. Gos. Univ., Ser. 1, Fiz. Mat. Inf., No. 2, 28-30 (2000).

28. V. A. Svetlichnyi, T. N. Kopylova, G. V. Maier, and I. N. Lapin, Izv. Vyssh. Uchebn. Zaved., Fiz., 48, No. 9, 12-16 (2005).

29. M. Shiek-Bahae, A. Said, and E. Van Stryland, Opt. Lett., 14, No. 17, 955-957 (1989).

30. E. S. Voropai and M. P. Samtsov, Opt. Spektrosk., 62, No. 1, 64-67 (1987).

31. B. P. Nikol'skii, ed., Handbook of Chemistry [in Russian], Vol. 1, Khimiya, Leningrad (1971).

32. E. S. Voropai, A. P. Lugovskii, V. I. Popechits, and M. P. Samtsov, Dokl. Akad. Navuk BSSR, 30, No. 3, 230232 (1986). 